\title{
Synthesis, Confirmation and Characterization of MnS Nanocomposites
}

\author{
Shabna .S \\ Department of Physics, Sree Narayana College Chengannur
}

\begin{abstract}
The nanoparticles and nanostructured materials have attracted great interest because their properties such as quantum confinement of electrons and holes, surface effects and geometrical confinement of phonons are different from those of bulky materials. Nano particles have a rather large number of atoms, but its size is comparable with characteristics dimensions describing the behavior of electrons and holes, thus creating an intermediate regime between molecules and bulk crystals. The optical responses of metal nanoparticles, nanoapertures in metal films and Meta materials are focuses on enhancing local electromagnetic fields to enormous facilitate light matter interactions. This work focuses on the synthesis and confirmation of MnS nanocomposites.
\end{abstract}

\section{Introduction}

Nanomaterials are cornerstones of nanoscience and nanotechnology. At the nanoscale, materials begin to exhibit distinct properties that affect biological, chemical and physical behaviors. These materials, notable for their extremely small feature size, have the potential for wide ranging industrial, biomedical and electronic applications.

The realm of nanotechnology is not new. In medieval age churches, nanoparticles were incorporated in the manufacturing and designing of the window glasses and each size corresponds to particular characteristic colours such as green, red, orange etc. Einstein in his post doctoral studies calculated the size of a sugar particle as $1 \mathrm{~nm}$. So that Einstein and glassworkers in the medieval age can be considered as nanoscientists. What's new is to discover right tools for this job. Metal nano particles in colloidal solution can be prepared by lots of methods such as laser ablation, chemical reaction or co-precipitation method etc.

\section{Synthesis of MnS Nanoparticles}

Uniform particles are usually prepared via homogeneous precipitation reaction. Chemical precipitation method deals with growing nanoparticles of inorganic materials through chemical reaction of their precursors. By slowing the reaction temperature or using suitable stabilizing agents, the growth rate of the particle and its particle size can be controlled. Often suitable capping agents are used to achieve the desired grain size.

$\mathrm{MnS}$ nanoparticles of present study are prepared through simple chemical precipitation method using Manganese Chloride as precursor. The principle involved in this technique is the precipitation of metal ions with Chloride ions in the solution. 0.1 M aqueous solution of Manganese Chloride and $0.1 \mathrm{M}$ aqueous solution of Sodium Sulphide were mixed in the presence of EDTA as capping agent. First solutions of $50 \mathrm{ml} \mathrm{MnCL}_{2}$ and $50 \mathrm{ml} \mathrm{Na}_{2} \mathrm{~S}$ were prepared in double distilled water. $10 \mathrm{ml}$ of $\mathrm{MnCl}_{2}$ and $5 \mathrm{ml}$ of EDTA were mixed together and stirred for 30 minutes on a magnetic stirrer to get a homogeneous solution. This was followed by drop wise addition of $10 \mathrm{ml}$ of $\mathrm{Na}_{2} \mathrm{~S}$ under vigorous stirring for 1 hour. A chocolate brown colour precipitate was obtained which was separated by centrifugation and washed up to 6 times with double distilled water and finally with acetone. The precipitate was then dried and powdered.

\section{Experimental Techniques}

UV-Visible Absorption Studies of MnS Nanoparticle Ultra violet - visible Spectroscopy is widely used for establishing the quantum confinement effect in nanostructured materials. UV-VIS absorption spectra can also be used for calculating the optical band gap of semiconductor nanomaterials. Ultra violet spectroscopy involves the spectroscopy of photons in the UV region. Ultra violet spectroscopy deals with the recording of absorption of light in the visible and ultraviolet region of the spectrum.

Most of the spectrometers record wavelength versus absorbance. The absorbance of the sample dispersed in the medium and absorbance co-efficient is estimated from the Beer-Lambert Law and is given by

$$
T=\frac{I}{I_{0}}=10^{-\alpha l}=10^{-\varepsilon l c}
$$

Where ' $\mathrm{T}$ ' is the transmittivity, ' $\alpha$ ' is the absorption coefficient of the substance, ' $l$ ' is the distance the light travels through the material, ' $\varepsilon$ ' is the molar absorptivity of the absorber and ' $\mathrm{c}$ ' is the molar concentration. ' $\mathrm{I}$ ' and ' $I_{0}$ ' are the intensities of incident and transmitted light respectively.

Nanoparticles of $\mathrm{MnS}$ were dispersed in water and ultrasonicated to record the absorption spectrum of asprepared nanostructured MnS. The optical absorption spectra of the nanoparticles of $\mathrm{MnS}$ were recorded at room temperature in the wavelength region from 190-900nm using ELICO SL 159 spectrophotometer.

The exact value of the band gap can be determined by plotting $(\alpha h v)^{1 / n}$ versus ' $h v$ ' graph and extrapolating the straight line portion of the graph to ' $h v$ ' axis.

\section{X-Ray Diffraction Study of MnS Nanoparticles}

$\mathrm{XRD}$ is a non destructive and highly quantitative powerful tool for the structural characterization of nanosize materials. The wavelength of X-rays is on the atomic scale making Xray diffraction a primary tool for probing structure of nanomaterials. X-ray diffractograms of nanomaterials 


\section{International Journal of Science and Research (IJSR) \\ ISSN (Online): 2319-7064 \\ Index Copernicus Value (2016): 79.57 | Impact Factor (2015): 6.391}

provide a wealth of information on phase, composition, crystalline size, lattice strain etc. The small grain size of the clusters in nanophase materials leads to the line broadening in X-ray diffraction pattern.

The width of the diffraction peak is determined by the number of planes in the crystalline and the dynamical diffraction theory of perfect crystals predicts the existence of very sharp diffraction lines, in which there may be only a small inherent broadening due to the uncertainty principle. Scherrer used this principle to obtain a relationship between line broadening and the particle size. Scherrer equation is given by,

$$
D=0.9 \lambda / \beta \cos \theta
$$

Where ' $\mathrm{D}$ ' is the diameter of the crystal, ' $\lambda$ ' is the wavelength of X-ray and ' $\beta$ ' is the FWHM

In the present work, X-ray diffraction pattern of the as prepared $\mathrm{MnS}$ samples are recorded using a Philips Xpertpro Diffractometer and using $C u k \alpha$ radiation over the diffraction

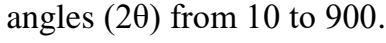

\section{Result and Discussion}

\section{UV-Visible Absorption Spectrum of Nanosize MnS}

The UV-Visible absorption spectrum and plot of $(\alpha h v)^{2}$ vs $\mathrm{h} v$ is of nanoparticles of $\mathrm{MnS}$ of present study are shown

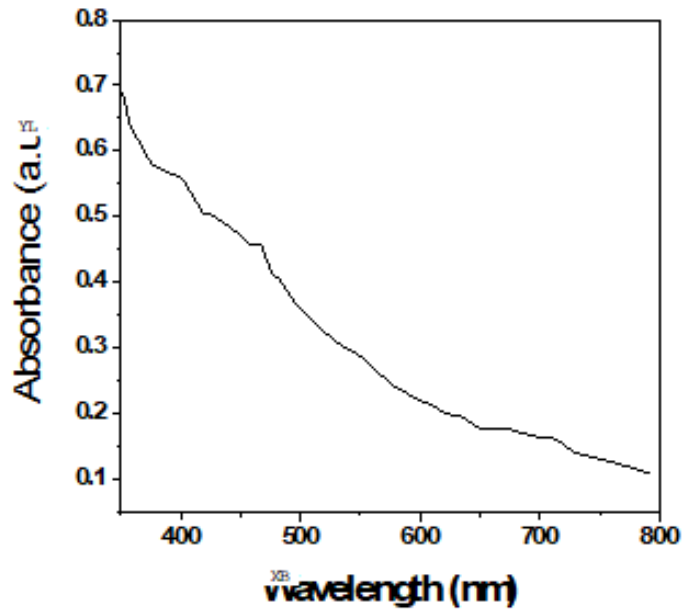

UV-Visible absorption spectrum of MnS nanoparticles

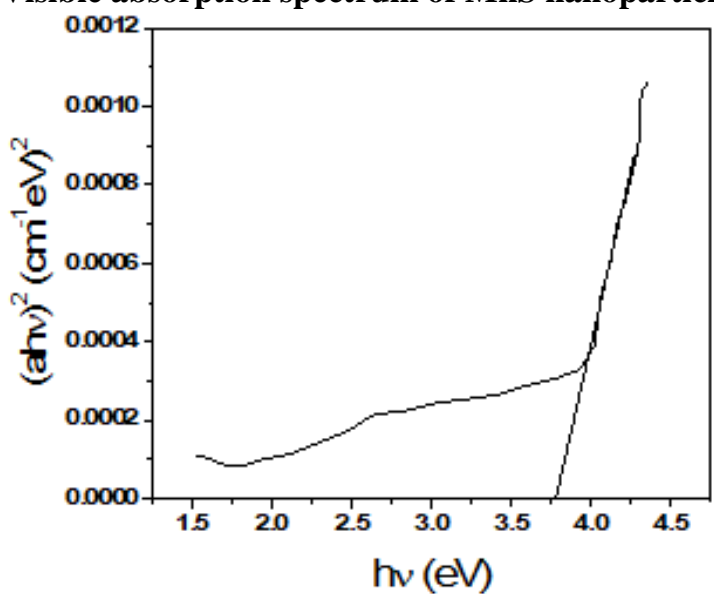

Plot of ahv vs hv of MnS nanoparticles

The band gap of nanoparticles of $\mathrm{MnS}$ as calculated from above figure was found to be $3.6 \mathrm{eV}$. This value is high compared to the band gap value of bulk $\mathrm{MnS}$, ie, $3.1 \mathrm{eV}$. This increase in band gap value of nanoparticles of $\mathrm{MnS}$ of present study can be attributed to quantum confinement effect.

\section{X-RAY Diffraction Studies of MnS Nanoparticles}

Figure shows diffraction peaks at $2 \theta$ values of $22.9^{0}, 32.7^{0}$, $44.5^{\circ}, 50.5^{\circ}$ and $60.6^{\circ}$ corresponding to $\mathrm{d}$ values of 3.87 , $2.73,2.03,1.81$ and $1.52 \AA$ respectively. The peaks are identified to originate from (100), (110), (200), (220) and (212) planes of the hexagonal phase of MnS (JCPDS Card No. 40-1289).

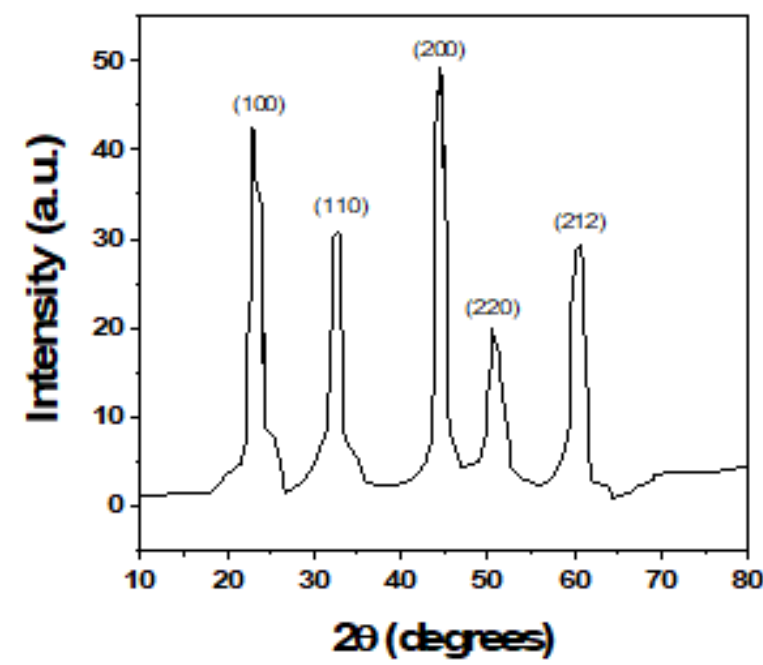

XRD pattern of MnS nanoparticles

The broadening of the diffraction peaks in figure indicates the nanocrystalline nature of the sample. Line broadening in the XRD pattern of nanoparticles is a good criterion to use in nanostructure analysis.

The grain size of the as prepared MnS nanoparticles of the present study is calculated by using the Scherrer's equation and is obtained as approximately $8 \mathrm{~nm}$.

\section{Conclusion}

Manganese Sulphide nanoparticles have interesting chemical and physical properties suitable for technological applications. The direct and wide band gap and the polymorphism behavior of the MnS crystals make them as appropriate for different kinds of semiconductor optical devising. In the present work, nanoparticles of $\mathrm{MnS}$ are prepared by co-precipitation method. X-ray Diffraction Pattern of the samples was recorded and the grain sizes calculated from Debye-Scherrer equation was found to be approximately $8 \mathrm{~nm}$. The band gap of the nanoparticles of $\mathrm{MnS}$ was calculated from the UV-Visible absorption spectrum and was found to be $3.6 \mathrm{eV}$. The result suggests that $\mathrm{MnS}$ nanoparticles are applicable in optical detector laser, sensor, imaging, photo electrochemistry, photocatalysis and biomedicine. 


\section{International Journal of Science and Research (IJSR)}

ISSN (Online): 2319-7064

Index Copernicus Value (2016): 79.57 | Impact Factor (2015): 6.391

\section{References}

[1] G Cao, "Nanostructures and nanomaterials-synthesis, properties and Applications", (2004) Imprial College Press London.

[2] K R Jain,"Physics of Semiconductor Nanostructures", Narosa Publications 1999.

[3] A. Uma Maheswari, S Saravana Kumar and M.Sivakumar, J. NanoSci.Nano Tecnol.13(2013)4409

[4] A.R Wilkinson and R.G Elliman, J.Appl. Phys.96,(2004)4018

[5] S.J. Chipera and D.L. Bish, Advances in X-Ray analysis.34 (1991)473.

Volume 6 Issue 12, December 2017

www.ijsr.net

Licensed Under Creative Commons Attribution CC BY 